\title{
NILPOTENT MINIMUM LOGIC NM AND PRETABULARITY
}

\begin{abstract}
This paper deals with pretabularity of fuzzy logics. For this, we first introduce two systems $\mathrm{NM}^{n f p}$ and $\mathrm{NM}^{\frac{1}{2}}$, which are expansions of the fuzzy system NM (Nilpotent minimum logic), and examine the relationships between $\mathrm{NM}^{n f p}$ and the another known extended system $\mathrm{NM}^{-}$. Next, we show that $\mathrm{NM}^{n f p}$ and $\mathrm{NM}^{\frac{1}{2}}$ are pretabular, whereas NM is not. We also discuss their algebraic completeness.

Keywords: Pretabularity, nilpotent minimum logic, algebraic semantics, fuzzy logic, finite model property.
\end{abstract}

\section{Fuzzy logic and pretabularity}

This paper is a contribution to the study of pretabularity of fuzzy logics. In general, a logic L is said to be pretabular if it does not itself have a finite characteristic matrix (algebra, or frame), but every normal extension of it does (see $[4,7,8,11,13]$ ). Note that Dunn (and Meyer) $[3,5]$ investigated the pretabularity of the semi-relevance logic RM (R with mingle) and the Dummett-Gödel logic G. One interesting fact is that these systems can be also regarded as fuzzy logics. ${ }^{1}$ Then, a natural question is now raised as follows.

\footnotetext{
${ }^{1}$ According to Cintula (and Běhounek) [1, 2], a (weakly implicative) logic L is said to be fuzzy if it is complete with respect to (w.r.t.) linearly ordered matrices (or algebras) and core fuzzy if it is complete w.r.t. standard algebras (i.e., algebras on the real unit interval $[0,1])$.
} 
Which fuzzy logics are pretabular?

This question, on the one hand, is not interesting in the sense that most basic fuzzy logics such as UL (Uninorm logic), MTL (Monoidal t-norm logic), and BL (Basic fuzzy logic) are not pretabular because such logics have some axiomatic extensions (henceforth, extensions for short) without finite characteristic matrices. On the other hand, it is interesting in that while, since then, no further pretabular fuzzy logics have been introduced, we can still introduce other concrete fuzzy logic systems.

We introduce two new pretabular systems as fuzzy logics, which we shall call the fixed-pointed nilpotent minimum logic $\mathrm{NM}^{\frac{1}{2}}$ and the nonfixed-pointed nilpotent minimum logic $\mathrm{NM}^{n f p}$. These two are the systems expanding and extending, respectively, the well-known fuzzy system NM (Nilpotent minimum logic) [6]. ${ }^{2}$ In particular, the system $\mathrm{NM}^{n f p}$ can be regarded as a Hilbert-style presentation of $\mathrm{NM}^{-}$(the NM with (BP) below), which is one of the extensions of NM introduced in $[9,10]$. For this purpose, we first introduce these two systems and examine the relationship between $\mathrm{NM}^{n f p}$ and $\mathrm{NM}^{-}$. We then show that $\mathrm{NM}^{n f p}$ and $\mathrm{NM}^{\frac{1}{2}}$ are pretabular while NM is not. We also discuss their algebraic completeness.

\section{Nilpotent minimum logics}

The nilpotent minimum logic NM can be based on a countable propositional language with formulas $F m$ built inductively as usual from a set of propositional variables $V A R$, binary connectives $\rightarrow, \&, \wedge$, and constant $\mathbf{F}$, with defined connectives: (df1) $\neg A:=A \rightarrow \mathbf{F}$; (df2) $A \vee B:=((A \rightarrow B) \rightarrow$ $B) \wedge((B \rightarrow A) \rightarrow A) ;($ df 3$) A \leftrightarrow B:=(A \rightarrow B) \wedge(B \rightarrow A)$.

The constant $\mathbf{T}$ is defined as $\mathbf{F} \rightarrow \mathbf{F}$. For the rest of this paper, we use the customary notations and terminology, and the axiom systems to provide a consequence relation.

We start with the following axiomatizations of NM and its two expansions.

DEFinition 1.

(i) ([6]) $\mathrm{NM}$ consists of the following axiom schemes and rules: A1. $(A \rightarrow B) \rightarrow((B \rightarrow C) \rightarrow(A \rightarrow C))$;

${ }^{2}$ For the definitions of expansion and extension, see Definition 9 in [2]. 
A2. $(A \& B) \rightarrow A$;

A3. $(A \& B) \rightarrow(B \& A)$;

A4. $(A \wedge B) \rightarrow A$

A5. $(A \wedge B) \rightarrow(B \wedge A)$;

A6. $(A \&(A \rightarrow B)) \rightarrow(A \wedge B)$;

A7. $(A \rightarrow(B \rightarrow C)) \leftrightarrow((A \& B) \rightarrow C)$;

A8. $((A \rightarrow B) \rightarrow C) \rightarrow(((B \rightarrow A) \rightarrow C) \rightarrow C)$;

A9. $\mathbf{F} \rightarrow A$

A10. $\neg \neg A \rightarrow A ;$

A11. $((A \& B) \rightarrow \mathbf{F}) \vee((A \wedge B) \rightarrow(A \& B))$;

$A \rightarrow B, A \vdash B$ (modus ponens, mp);

$A, B \vdash A \wedge B$ (adjunction, adj).

(ii) - Non-fixed-pointed nilpotent minimum logic $\mathrm{NM}^{n f p}$ is NM plus $(A \vee \neg A) \rightarrow((A \& A) \vee(\neg A \& \neg A))$ (Non-fixed-point, Nfp).

- Fixed-pointed nilpotent minimum logic $\mathrm{NM}^{\frac{1}{2}}$ is NM plus $\frac{\overline{1}}{2}$ and $\overline{\frac{1}{2}} \leftrightarrow \neg \overline{\frac{1}{2}}$ (Fixed-point, Fp). ${ }^{3}$

For convenience, ' $\neg$, ' $\wedge$,' ' $\vee$,' and ' $\rightarrow$ ' are used ambiguously as propositional connectives and as algebraic operators, but context should clarify their meaning. follows.

The algebraic counterpart of $\mathrm{L} \in\left\{N M, N M^{n f p}, N M^{\frac{1}{2}}\right\}$ is defined as

Definition 2.

(i) An NM-algebra is a structure $\mathcal{A}=(A, \top, \perp, \wedge, \vee, *, \rightarrow, \neg)$, where $\neg x:=x \rightarrow \perp$ for all $x \in A$ and $x \vee y:=((x \rightarrow y) \rightarrow y) \wedge((y \rightarrow x) \rightarrow$ $x)$ for all $x, y \in A$, such that:

- $(A, \top, \perp, \wedge, \vee)$ is a bounded lattice with top element $\top$ and bottom element $\perp$;

- $(A, *, \top)$ is an integral commutative monoid;

$-y \leq x \rightarrow z$ iff $x * y \leq z$ (residuation);

$-\top^{-}=(x \rightarrow y) \vee(y \quad \rightarrow \quad x) \quad$ (prelinearity);

\footnotetext{
${ }^{3}$ The constant $\frac{\overline{1}}{2}$ does not necessarily correspond to the actual fraction $\frac{1}{2}$. Since the standard negation $\neg x$ is defined as $1-x$ in $[0,1]$ and $\frac{1}{2}$ has the role of fixed-point in that $\frac{1}{2}=\neg \frac{1}{2}$ in $[0,1], \frac{1}{2}$ is used as a representative of fixed-point. Therefore, here we use $\frac{1}{2}$ as the constant for denoting a fixed-point element of any algebra.
} 
- $\neg \neg x=x$ (involution);

- $\mathrm{\top}=((x * y) \rightarrow \perp) \vee((x \wedge y) \rightarrow(x * y))$ (weak nilpotent minimum).

(ii) - An $N M^{n f p}$-algebra is an NM-algebra satisfying $x \vee \neg x \leq(x *$ $x) \vee(\neg x * \neg x)$ (non-fixed-point).

- An $N M^{\frac{1}{2}}$-algebra is an NM-algebra with $\frac{1}{2}$ satisfying $\frac{1}{2}=\neg \frac{1}{2}$ (fixed-point).

Consider the system $\mathrm{NM}^{-}$, which is NM plus $(B P) \neg(\neg(A \& A)$ \& $\neg(A \& A)) \leftrightarrow(\neg(\neg A \& \neg A) \& \neg(\neg A \& \neg A))$. This system was introduced as the logic with semantics on $[0,1]$ minus the fixed-point in [9]. Let linearly ordered algebras be chains. We finally consider the relationships between $\mathrm{NM}^{n f p}$ and $\mathrm{NM}^{-}$.

\section{THEOREM 1.}

(1) ([9]) A nontrivial NM-chain satisfies $\left(B P^{\mathcal{A}}\right) \neg(\neg(x * x) * \neg(x * x))=$ $\neg(\neg x * \neg x) * \neg(\neg x * \neg x)$ iff it does not contain a fixed-point.

(2) A nontrivial NM-chain satisfies (non-fixed-point) iff it does not contain a fixed-point.

Proof: For the left-to-right direction of (2), we assume that there is an element $x>\perp$ such that $x=\neg x$ and show that $x \vee \neg x>(x * x) \vee(\neg x * \neg x)$. Let $x=\neg x$. Then, since $x * x=\neg x * \neg x=\perp$, we have that $x \vee \neg x>$ $(x * x) \vee(\neg x * \neg x)=\perp$. For the right-to-left direction of (2), assume that $x \neq \neg x$ for all $x \in A$. First, consider the case $x<\neg x$. Using (weak nilpotent minimum), we can obtain that $x * x=\perp$ and $\neg x * \neg x=\neg x$ and thus $x \vee \neg x=\neg x=(x * x) \vee(\neg x * \neg x)$; therefore, $x \vee \neg x \leq(x * x) \vee(\neg x * \neg x)$. Consider the case $\neg x<x$. Its proof is analogous to that of the case $x<\neg x$.

COROLlary 1. A nontrivial NM-chain satisfies $\left(B P^{\mathcal{A}}\right)$ iff it satisfies (nonfixed-point).

Now consider the systems $\mathrm{NM}^{n f p}$ and $\mathrm{NM}^{-}$synthetically. We can show the following.

THEOREM 2. The system $N M^{n f p}$ proves:

$(B P) \neg(\neg(A \& A) \& \neg(A \& A)) \leftrightarrow(\neg(\neg A \& \neg A) \& \neg(\neg A \& \neg A))$.

Proof: First, note that the following are theorems of NM: (a) $(A \rightarrow B) \vee$ $(B \rightarrow A)$; (b) $A \rightarrow(\neg A \rightarrow B)$; (c) $A \rightarrow(B \rightarrow A) ;(\mathrm{d}) \neg \neg A \leftrightarrow A$; (e) $((A \& A) \vee(\neg A \& \neg A)) \rightarrow(A \vee \neg A)$. 
$(\Rightarrow)$ 1. $(A \rightarrow \neg A) \vee(\neg A \rightarrow A)(\mathrm{a})$;

2. $((A \rightarrow \neg A) \&(A \rightarrow \neg A)) \vee((\neg A \rightarrow A) \&(\neg A \rightarrow A))(1, \mathbf{T} \& \mathbf{T} \leftrightarrow$ T);

3. $((A \rightarrow \neg A) \&(A \rightarrow \neg A)) \rightarrow(\neg((A \rightarrow \neg A) \&(A \rightarrow \neg A)) \rightarrow$ $((\neg A \rightarrow A) \&(\neg A \rightarrow A)))(\mathrm{b})$;

4. $\quad((\neg A \rightarrow A) \&(\neg A \rightarrow A)) \rightarrow(\neg((A \rightarrow \neg A) \&(A \rightarrow \neg A)) \rightarrow$ $((\neg A \rightarrow A) \&(\neg A \rightarrow A)))(\mathrm{c})$;

5. $\neg((A \rightarrow \neg A) \&(A \rightarrow \neg A)) \rightarrow((\neg A \rightarrow A) \&(\neg A \rightarrow A))(2,3,4$, adj, mp);

6. $\neg(\neg(A \& A) \& \neg(A \& A)) \rightarrow(\neg(\neg A \& \neg A) \& \neg(\neg A \& \neg A))(5, \mathrm{~d},(\mathrm{df} 4)$ $A \& B:=\neg(A \rightarrow \neg B))$.

$(\Leftarrow)$ 1. $(A \vee \neg A) \leftrightarrow((A \& A) \vee(\neg A \& \neg A))$ (e, Nfp, adj, df3);

2. $\neg(A \vee \neg A) \leftrightarrow \neg((A \& A) \vee(\neg A \& \neg A)) \leftrightarrow \mathbf{F}$ (1, df1, A9, adj);

3. $(A \wedge \neg A) \leftrightarrow(\neg(A \& A) \wedge \neg(\neg A \& \neg A)) \leftrightarrow \mathbf{F}(2, \mathrm{~d}$, De Morgan);

4. $(\neg(A \& A) \& \neg(A \& A)) \wedge(\neg(\neg A \& \neg A) \& \neg(\neg A \& \neg A)) \leftrightarrow \mathbf{F}(3, \mathbf{F} \& \mathbf{F} \leftrightarrow$

F);

5. $\neg(\neg(A \& A) \& \neg(A \& A)) \vee \neg(\neg(\neg A \& \neg A) \& \neg(\neg A \& \neg A)) \leftrightarrow \mathbf{T}(4$, $\neg \mathbf{F} \leftrightarrow \mathbf{T}$, De Morgan);

6. $\quad(\neg(\neg A \& \neg A) \& \neg(\neg A \& \neg A)) \quad \rightarrow \quad \neg(\neg(A \& A) \& \neg(A \& A)) \quad(4, \quad 5$, Boolean property).

Then, from Theorem 2, the following question arises when we just think of the systems synthetically.

- Open Problem: Does the system $\mathrm{NM}^{-}$prove $(N f p)$ synthetically?

According to Corollary 1, it seems possible to show this since the conditions $\left(B P^{\mathcal{A}}\right)$ and (non-fixed-point) both correspond to the condition 'no fixedpoint.' However, we have not yet proved this. To the author, it seems that the correct axiomatization of the extension of $\mathrm{NM}$ with the semantics on $[0,1]^{-}$, i.e., $[0,1] \backslash\left\{\frac{1}{2}\right\}$, is not the axiomatization of $\mathrm{NM}^{-}$, but that of $\mathrm{NM}^{n f p}$.

\section{Pretabularity}

For $\mathrm{L} \in\left\{N M, N M^{n f p}, N M^{\frac{1}{2}}\right\}$, by an L-algebra, we henceforth denote any

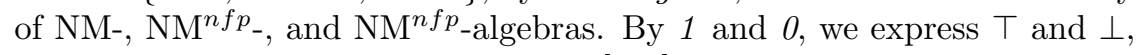
respectively, on the real unit interval $[0,1]$ or on a subset of it with top 
and bottom elements 1,0. We refer to L-algebras on such a carrier set as $S^{L}$-algebras. $\mathrm{S}^{L}$-algebras are defined as follows:

Definition 3. The operations for an $S^{L}$-algebra are defined as follows.

(1) ([6]) Let the carrier set $S$ be $[0,1]$. An $S^{N M}$-algebra is an algebra satisfying: T1. $x \wedge y=\min (x, y)$; T2. $x \vee y=\max (x, y)$; T3. $x \rightarrow y=1$ if $x \leq y$, and otherwise $x \rightarrow y=\max (1-x, y)$; T4. $\neg x=1-x .^{4}$

(2) Let the carrier set $S$ be a subset of [0,1] with top and bottom elements 1,0 .

- An $S^{N M^{n f p}}$-algebra is an $S^{N M}$-algebra whose carrier set $S$ has no fixed-point.

- An $S^{N M \frac{1}{2}}$-algebra is an $S^{N M}$-algebra whose carrier set $S$ has $\frac{1}{2}$, a fixed-point.

By $S_{[0,1]}^{L}$-algebra, we henceforth denote the $S^{L}$-algebra on $[0,1]$; by $S_{[0,1]^{-}}^{L}$-algebra, the $S^{L}$-algebra on $[0,1] \backslash\left\{\frac{1}{2}\right\}$; by $S_{n}^{L}$-algebra, the $S^{L}$-algebra whose elements are in $\left\{0, \frac{1}{n-1}, \ldots, \frac{n-2}{n-1}, 1\right\}$. Generalizing, $S$-algebra refers to any algebra whose elements form a chain with the greatest and least elements, and whose operations are defined in an analogous way.

Note that $S$-algebras having $\frac{1}{2}$ as an element $x$ such that $x=\neg x$ are said to be fixed-pointed, and otherwise non-fixed-pointed. A logic L is said to be fixed-pointed if $\mathrm{L}$ is characterized by an $S$-algebra having a fixedpoint, and otherwise is non-fixed-pointed. An extension of $\mathrm{L}$ is said to be proper if it does not have exactly the same theorems as L.

DEFINITION 4.

(i) (Tabularity) A logic L is tabular if $\mathrm{L}$ has some finite characteristic algebra.

(ii) (Pretabularity) A logic L is pretabular if (a) L is not tabular and (b) every proper extension of $\mathrm{L}$ has some finite characteristic algebra.

Now, we show that $\mathrm{L} \in\left\{N M^{n f p}, N M^{\overline{\frac{1}{2}}}\right\}$ is pretabular, but the systems $\mathrm{NM}$ is not. We first introduce some known pretabular logics.

FACT 1. ([3, 5]) Each of RM and G is pretabular.

\footnotetext{
${ }^{4}$ In general, the involutive negation is defined as the negation $n$ satisfying $n(n(x))=x$ for all $x \in[0,1]$. Since any involutive negation $[0,1]$ can be isomorphic to $1-x$, for convenience, we take this definition.
} 
We then divide the work into a number of propositions following the line in $[3,5]$.

Proposition 1. Let $\mathcal{X}$ be an extension of $L \in\left\{N M^{n f p}, N M^{\frac{1}{2}}\right\}, \mathcal{A}$ be an $\mathcal{X}$-algebra, and $a \in \mathcal{A}$ be such that $a<T$. Then, there is a homomorphism $h$ of $\mathcal{A}$ onto an $S$-algebra which is an $\mathcal{X}$-algebra, such that $h(a)<1$.

Proof: The proof is analogous to Theorem 3 in [3] and Theorem 11.10.4 in [4].

\section{Proposition 2.}

(i) Let L be the system $\mathrm{NM}^{n f p}$. Let $S_{1}^{L}, S_{2}^{L}, S_{4}^{L}, S_{6}^{L}, \ldots$, i.e., $S_{1}^{L}$ and $S_{2 n}^{L}, 1 \leq n \in N$, be the sequence of $S^{L}$-algebras relabeled in order as $M_{1}^{L}, M_{2}^{L}, M_{3}^{L}, \ldots$ If a sentence $A$ is valid in $M_{i}^{L}$, then $A$ is valid in $M_{j}^{L}$, for all $j, j \leq i$.

(ii) Let L be the system $\mathrm{NM}^{\frac{1}{2}}$. Let $S_{1}^{L}, S_{3}^{L}, S_{5}^{L}, S_{7}^{L}, \ldots$, i.e., $S_{2 n-1}^{L}, 1 \leq n$ $\in N$, be the sequence of $S^{L}$-algebras relabeled in order as $M_{1}^{L}, M_{2}^{L}$, $M_{3}^{L}, \ldots$. If a sentence $A$ is valid in $M_{i}^{L}$, then $A$ is valid in $M_{j}^{L}$, for all $j, j \leq i$.

Proof: Since each $S_{j}^{L}$ is (isomorphic to) a subalgebra or a homomorphic image of $S_{i}^{L}$, (i) and (ii) are immediate.

Proposition 3. In $S^{N M}$-algebras, when $i$ is even $(\geq 4), S_{i}^{N M}$ validates a sentence $A$ that is not valid in any odd-valued $S_{j}^{N M}, 3 \leq j \leq i$.

Proof: The claim can be verified by considering the sentence (Nfp), which is valid in every even-valued $S_{i}^{N M}$, but not in $S_{3}^{N M}$ (and thus not in any odd-valued $S_{j}^{N M}, j \geq 3$ ).

Remark 1. Proposition 3 implies that every valid sentence in $S_{[0,1]}^{N M}$ must be valid in $S_{[0,1]^{-}}^{N M}$, but there is a valid sentence in $S_{[0,1]^{-}}^{N M}$ that is not in $S_{[0,1]}^{N M}$.

Now, we recall the concept of a Lindenbaum-Tarski algebra. Let $\mathrm{L} \in$ $\left\{N M^{n f p}, N M^{\frac{1}{2}}\right\}$ and $T$ be a theory in L. We define $[A]=\left\{B: T \vdash_{L} A \leftrightarrow\right.$ $B\}$ and $\mathrm{L}=\{[A]: A \in F m\}$. The Lindenbaum-Tarski algebra Lind $_{T}$ w.r.t. $\mathrm{L}$ and $T$ is $\mathrm{L}$-algebra having the domain $\mathrm{L}$, operations $\#^{\mathrm{Lind}}\left(\left[A_{1}\right], \ldots,\left[A_{n}\right]\right)$ $=\left[\#\left(A_{1}, \ldots, A_{n}\right)\right]$, where $\# \in\{\wedge, \&, \rightarrow\}$, and the top and bottom elements 
are $[\mathbf{T}]$ and $[\mathbf{F}]$, respectively. We call this algebra the Lindenbaum-Tarski algebra $\mathcal{A}(L)$.

Where $\mathcal{X}$ is a propositional system and $\mathbf{V}$ is a set of atomic sentences, let $\mathcal{X} / \mathbf{V}$ be that propositional system like $\mathcal{X}$ except that its sentences contain no atomic sentences other than those in $\mathbf{V}$ and thus $\mathcal{A}(\mathcal{X} / \mathbf{V})$ be its corresponding Lindenbaum-Tarski algebra. The following is obvious.

Proposition 4. Let $\mathcal{X}$ be an extension of $L \in\left\{N M^{n f p}, N M^{\frac{\overline{1}}{2}}\right\}$. Then, $\mathcal{A}(\mathcal{X} / \boldsymbol{V})$ is an $\mathcal{X}$-algebra and is characteristic for $\mathcal{X} / \boldsymbol{V}$, since any nontheorem may be falsified under the canonical evaluation $v_{c}$, which sends every sentence $A$ to $[A]$, where $[A]$ is the set of all sentences $B$ such that $B \leftrightarrow A$.

Also, it follows from Propositions 1 and 4 that:

Proposition 5. Let $\mathcal{X}$ be an extension of $L \in\left\{N M^{n f p}, N M^{\frac{1}{2}}\right\}$. Then, if a sentence $A$ is not a theorem of $\mathcal{X}$, there is some $S^{L}$-algebra $S_{n}^{L}$ such that $S_{n}^{L}$ is an $\mathcal{X}$-algebra and $A$ is not valid in $S_{n}^{L}$.

Proof: If $A$ is not a theorem of $\mathcal{X}$, then, by Proposition $4, A$ is falsifiable in the $\mathcal{X}$-algebra $\mathcal{A}(\mathcal{X} / \mathbf{V})$, where $\mathbf{V}$ is the set of sentential variables occurring in $A$, by the canonical evaluation $v_{c}$. However, since $[A]$ is undesignated in $\mathcal{A}(\mathcal{X} / \mathbf{V})$, then, by Proposition 1 , there is a homomorphism $h$ of $\mathcal{A}(\mathcal{X} / \mathbf{V})$ onto an $S^{L}$-algebra $S^{L}$ such that $S^{L}$ is an $\mathcal{X}$-algebra and $h([A])<1$ in $S^{L}$. However, the composition of $h$ and $v_{c}, h \circ v_{c}(B)=h([B])$, is an evaluation that falsifies $A$ in $S^{L}$. Note that an $S^{L}$-subalgebra, the image $h(\mathcal{A}(\mathcal{X} / \mathbf{V})$, is finitely generated since it is the homomorphic image of $\mathcal{A}(\mathcal{X} / \mathbf{V})$, which is finitely generated by the elements $[p]$ such that $p \in \mathbf{V}$. Thus, this algebra is finitely generated by the elements $[p]$ such that $p \in \mathbf{V}$. It is obvious that every finitely generated $S^{L}$-subalgebra is finite and isomorphic to some $S_{n}^{L}$. Thus, this algebra is isomorphic to some $S_{n}^{L}$, which completes the proposition.

If $\mathcal{X}$ is $\mathrm{L}$ itself, we have the following completeness theorem as a corollary.

Corollary 2. (Completeness) For $L \in\left\{N M^{n f p}, N M^{\frac{1}{2}}\right\}$ and the set of $S^{L}$-algebras $\mathcal{S}^{\mathcal{L}}$, if a sentence $A$ is valid in $\mathcal{S}^{\mathcal{L}}$, then $A$ is a theorem of $L$.

Proof: By proposition 5, we have that if a sentence $A$ is not a theorem of L, there is some $S^{L}$-algebra $S_{n}^{L}$ such that $A$ is not valid in $S_{n}^{L}$. Thus, by contraposition, we obtain the claim. 
Finally, we turn to a proof of our principal results. THEOREM 3.

(i) $L \in\left\{N M^{n f p}, N M^{\frac{\overline{1}}{2}}\right\}$ is pretabular.

(ii) NM is not pretabular.

Proof: For (i), we show that every proper extension of $\mathrm{L}$ has a finite characteristic algebra. Let $M_{1}^{L}, M_{2}^{L}, M_{3}^{L}, \ldots$ be the sequence of $S^{L}$-algebras defined in Proposition 2. Let $I$ be the set of indices of those $S^{L}$-algebras that are $\mathcal{X}$-algebras, where $\mathcal{X}$ is the given proper extension of $\mathrm{L}$.

First, if $I$ contains an infinite number of indices, then $I$ contains every index because of Proposition 2. However, since every $S^{L}$-algebra $M_{i}^{L}$ is an L-algebra, it follows from Proposition 5 and Corollary 2 that $\mathcal{X}$ is identical with $\mathrm{L}$, which contradicts the hypothesis that $\mathcal{X}$ is a proper extension of $\mathrm{L}$.

Second, if I contains only a finite number of indices, then, by Proposition 2, there must be some index $i$ such that $I$ contains exactly those indices less then or equal to $i$. By construction, $S_{i}^{L}$ is an $\mathcal{X}$-algebra. Let a sentence $A$ not be a theorem of $\mathcal{X}$. Then, by Proposition $5, A$ is not valid in some $\mathcal{X}$-algebra $M_{h}^{L}$, and, by our choice of $i, h \leq i$. However, by Proposition 2, $A$ is not valid in $M_{i}^{L}$. Therefore, $M_{i}^{L}$ is the desired finite characteristic algebra.

$\mathrm{L}$ itself has no finite characteristic algebra, which can easily be shown by a proof similar to that of Sugihara in [12]. Therefore, it can be ensured that $\mathrm{L}$ is pretabular.

(ii) directly follows from (i), Proposition 3, and Remark 1. (Note that the system $\mathrm{NM}^{n f p}$ is a pretabular extension of NM.)

We finally remark some relationships between the results in Theorem 3 and algebraic results introduced in $[9,10]$.

Remark 2.

(1) The fact that $\mathrm{NM}^{n f p}$ is pretabular but NM is not can be algebraically obtained as a consequence of the full description of the lattice of subvarieties of the variety $\mathcal{N M}$ (see Theorems 2 and 3 and Figure 2 in [9] and Figure 1 in [10]).

(2) Pretabularity is a property related to logics whose associated varieties of algebras are locally finite. A variety of algebras is said to be locally finite if each of its finitely generated members is a finite algebras. We first note that the variety $\mathcal{N} \mathcal{M}$ is locally finite (see $[9,10])$. 
Thus, since the varieties $\mathcal{N} \mathcal{M}^{n f p}$ (the variety of non-fixed-pointed NM-algebras) and $\mathcal{N} \mathcal{M}^{\frac{1}{2}}$ (the variety of fixed-pointed NM-algebras) are subvarieties of $\mathcal{N} \mathcal{M}, \mathcal{N} \mathcal{M}^{n f p}$ and $\mathcal{N} \mathcal{M} \frac{\overline{1}}{2}$ are locally finite. These results show that every pretabular variety is locally finite, but not conversely.

\section{Concluding remarks}

We showed that the two fuzzy systems $\mathrm{NM}^{n f p}, \mathrm{NM}^{\frac{1}{2}}$ are pretabular while $\mathrm{NM}$ is not. We also showed that $\mathrm{NM}^{n f p}$ and $\mathrm{NM}^{-}$are semantically equivalent. However, we have not yet shown this syntactically. This problem should be addressed in future research. We also have another interesting question as follows: Let $\mathrm{L}_{1}$ and $\mathrm{L}_{2}$ be two pretabular logics complete w.r.t. characteristic algebras $S^{L_{1}}$ and $S^{L_{2}}$, and consider the logic L induced by the ordinal sum $S^{L_{1}} \oplus S^{L_{2}}$. Then, we can ask: Under which condition L is pretabular?

Acknowledgment. The author must thank the anonymous referees for their helpful comments. This work was supported by the Ministry of Education of the Republic of Korea and the National Research Foundation of Korea (NRF-2016S1A5A8018255).

\section{References}

[1] L. Běhounek and P. Cintula, Fuzzy logics as the logics of chains, Fuzzy Sets and Systems, Vol. 157 (2006), pp. 604-610.

[2] P. Cintula, Weakly Implicative (Fuzzy) Logics I: Basic properties, Archive for Mathematical Logic, Vol. 45 (2006), pp. 673-704.

[3] J. M. Dunn, Algebraic completeness for R-mingle and its extensions, The Journal of Symbolic Logic, Vol. 35 (1970), pp. 1-13.

[4] J. M. Dunn and G. Hardegree, Algebraic Methods in Philosophical Logic, Oxford University Press, Oxford, 2001.

[5] J. M. Dunn and R. K. Meyer, Algebraic completeness results for Dummett's $L C$ and its extensions, Mathematical Logic Quarterly, Vol. 17 (1971), pp. $225-230$. 
[6] F. Esteva and L. Godo, Monoidal t-norm based logic: towards a logic for left-continuous t-norms, Fuzzy Sets and Systems, Vol. 124 (2001), pp. 271-288.

[7] D. Gabbay and V. B. Shetman, Undecidability of modal and intermediate first-order logics with two individual variables, The Journal of Symbolic Logic, Vol. 58 (1993), pp. 800-823.

[8] L. Galminas and J. G. Mersch, A pretabular classical relevance logic, Studia Logica, Vol. 100 (2012), pp. 1211-1221.

[9] J. Gispert, Axiomatic extensions of the nilpotent minimum logic, Reports on Mathematical Logic, Vol. 37 (2003), pp. 113-123.

[10] C. Noguera, F. Esteva, and J. Gispert, On triangular norm based axiomatic extensions of the weak nilpotent minimum logic, Mathematical Logic Quarterly, Vol. 54 (2008), pp. 387-409.

[11] V. Rybakov, V. Kiyatkin, and M. Terziler, Independent bases for rules admissible in pretabular logics, Logic Journal of the Interest Group in Pure and Applied Logics, Vol. 7 (1999), pp. 253-266.

[12] T. Sugihara, Strict implication free from implicational paradoxes, Memoirs of the Faculty of Liberal Arts, Fukui University, Series 1, 1955, pp. $55-59$.

[13] K. Świrydowicz, There exists an uncountable set of pretabular extensions of the relevant logic $R$ and each logic of this set is generated by a variety of finite height, The Journal of Symbolic Logic, Vol. 73 (2008), pp. 1249-1270.

Jeonbuk National University

Department of Philosophy \& Institute of Critical Thinking and Writing Rm 417 Center for Humanities \& Social Sciences

Jeonju 54896, Korea

e-mail: eunsyang@jbnu.ac.kr 